\title{
A Rapidly Progressive Proptosis, a Case Report
}

\section{Afshin Tajdini, Mazen Sinjab, Somer Hassan}

Department of Ophthalmology, Al-Mouasat Hospital, Damascus, Syria.

Email: dr.tajdini@gmail.com

Received January $10^{\text {th }}, 2012$; revised February $16^{\text {th }}, 2012$; accepted March $8^{\text {th }}, 2012$

\begin{abstract}
In contrast to intraocular metastases, orbital metastases are a rare manifestation of systemic malignancies; such orbital metastases account for only $1 \%-13 \%$ of all orbital tumors [1]. Such tumors grow rapidly; they may undergo necrosis, as the rate of tumor angiogenesis cannot keep pace with the rapidly expanding mass. Here, we report a rare case of an orbital metastasis from a nasopharyngeal carcinoma.
\end{abstract}

Keywords: Proptosis; Orbital Metastasis; Nasopharyngeal Carcinoma; Undifferentiated Carcinoma

\section{Case History}

A 45-year-old heavy smoker male presented with a rapidly expanding left proptosis, diminished vision and restricted eye movements, which he had first noticed one month prior to presentation. The Left eye Examination revealed severe proptosis (grade IV), restricted eye movement in all directions (frozen eye), severe congested conjunctiva and clear medias. Tonometry was normal. The visual acuity was hand motion with no improvement on pinhole or correction. The fundus on the affected side showed normal disc, generalized RPE atrophy and macula showed dull foveal reflex. Retinal arteries were normal, while veins were congested, but there were no hemorrhages or exudates. There was a history of nasopharyngeal carcinoma (NPC) with metastasis to $3^{\text {rd }}$ and $4^{\text {th }}$ cervical spine and to the root of common carotid artery one year ago. He had received chemotherapy and radiotherapy treatment at the time of diagnosis. The last radiotherapy session was about 5 month before.

An orbital CT scan of the left orbit, that was done one month before, showed a large soft tissue mass $(5 \times 3 \mathrm{~cm})$ was extended from lateral wall of the orbit to the optic foramen and to the cranium, in order to lie between the frontal and temporal lobes. The mass pushed the glob to the medial and optic nerve to lateral. (Figure 1)

An orbital MRI of the left orbit showed a two-part large soft tissue mass $(5 \times 3 \mathrm{~cm} \mathrm{\&} 2 \times 3 \mathrm{~cm})$ that each part was connected to the other. The first bigger part $(5 \times$ $3 \mathrm{~cm}$ ) was extended from lateral wall of the orbit to the optic foramen and to the cranium, in order to lie between the frontal and temporal lobes. This part pushed the glob to the medial and optic nerve to lateral. The second part was smaller in size $(2 \times 3 \mathrm{~cm})$, extracranial, close to the lacrimal bone, with infiltration to temporal and masseter bone (Figure 2). MRI revealed the same T1 and T2 signal intensity with intermediate gadolinium enhancement, without any obvious invasion or pathological enhancement on brain parenchyma (Figure 3).

The tumoral cells on immunohistochemical staining were positive for cytokeratin (CK) (Figure 4), and negative for Leucocyte-Common-Antigen (LCA) (Figure 5), that were consistent with invasive, poorly differentiated, non-keratinizing Squamous cell carcinoma. The patient referred to an oncology center for receiving radiotherapy.

\section{Discussion}

Although nasopharyngeal carcinoma (NPC) commonly metastasizes to cervical lymph nodes, orbital metastasis

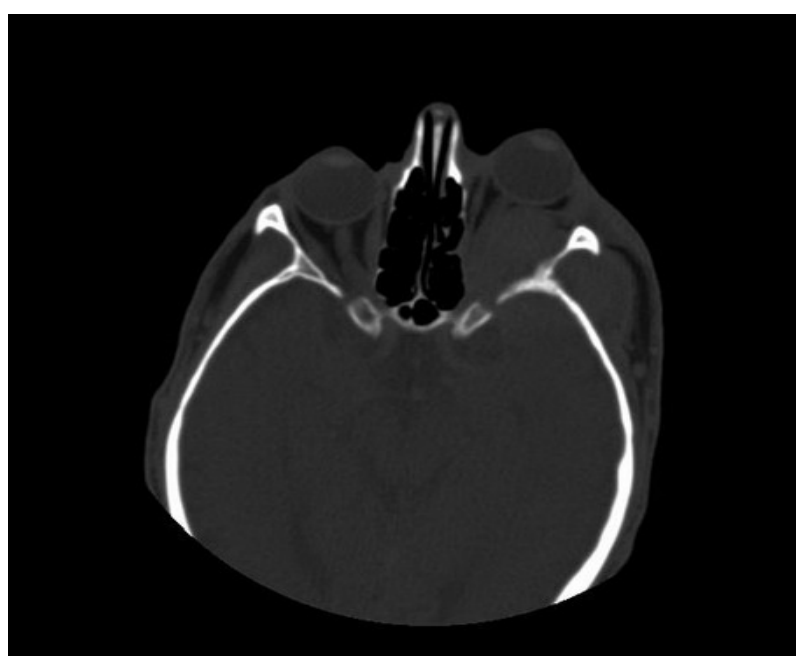

Figure 1. CT scan shows an orbital soft tissue mass, causing proptosis in a 45 years old man. 


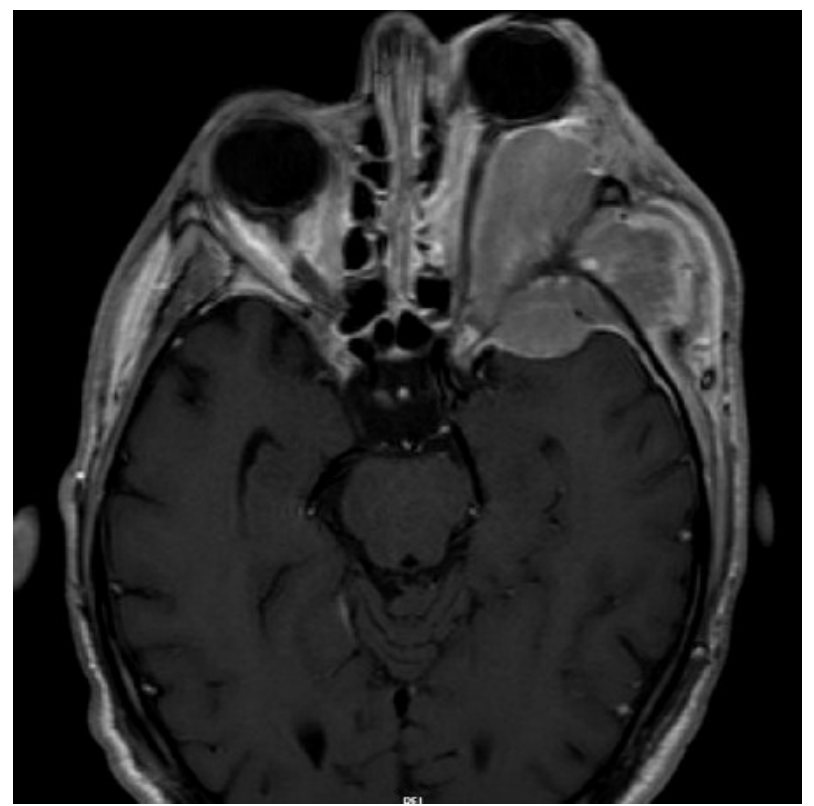

Figure 2. MRI shows a two-part large soft tissue mass that are connected together.

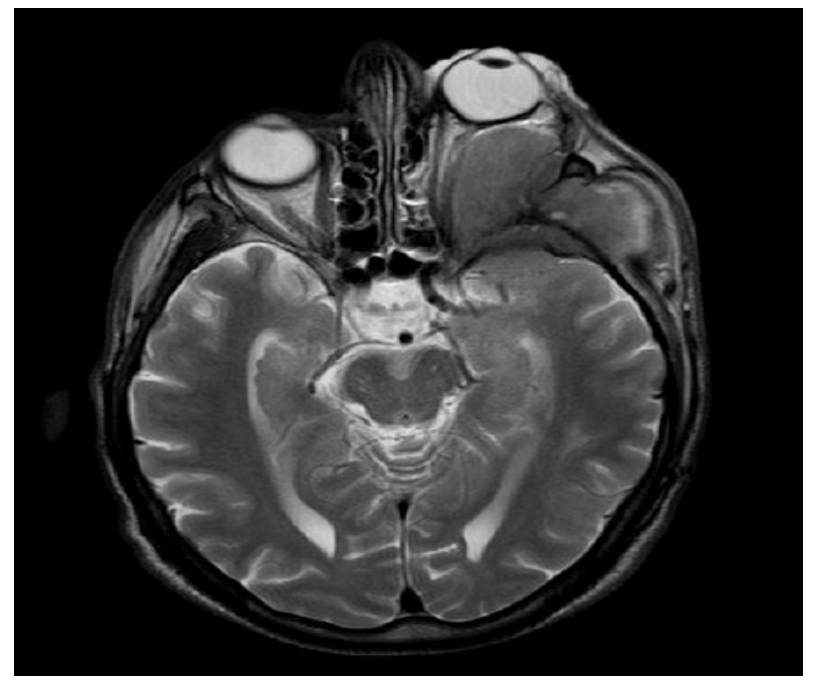

Figure 3. T2 imaging shows intermediate gadolinium enhancement, without obvious invasion on brain parenchyma.

are rare. Most cases of orbital involvement are through direct invasion typically via the cavernous sinus into the apex causing proptosis and muscle paralysis [2].

The mean age at time of presentation is 55 years. The primary tumor is most often a carcinoma involving the breast ( $40 \%)$, lung $(11 \%)$, or prostate ( $8 \%)$, followed by nasopharyngeal carcinoma. The most common manifestations of orbital metastases are diplopia, exophthalmos, inflammation, decreased visual acuity, pain, chemosis, and eyelid swelling, depending on the site affected. The symptoms usually occur acutely and progress rapidly, over the course of weeks to months [3,4].

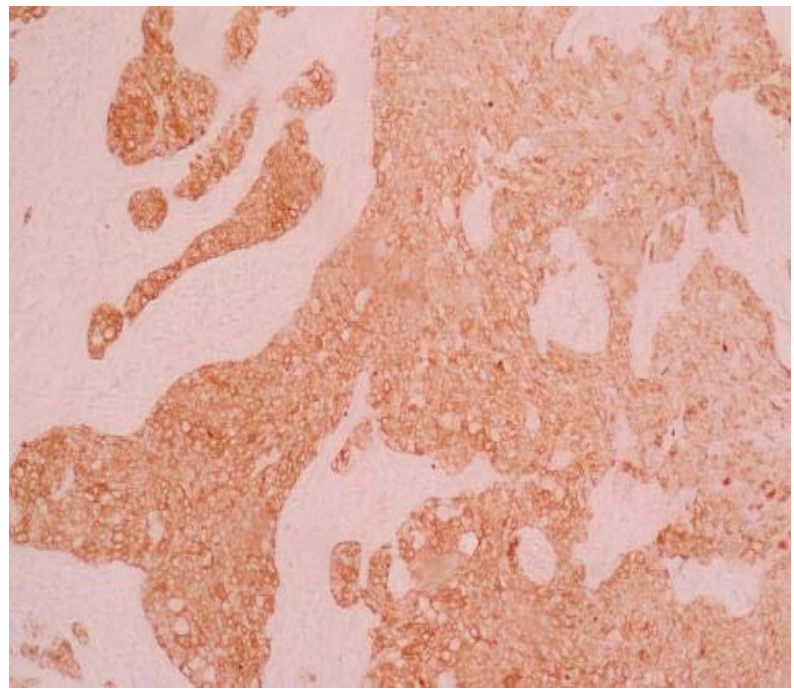

Figure 4. The tumoral cells on immunohistochemical staining were positive for cytokeratin (CK).

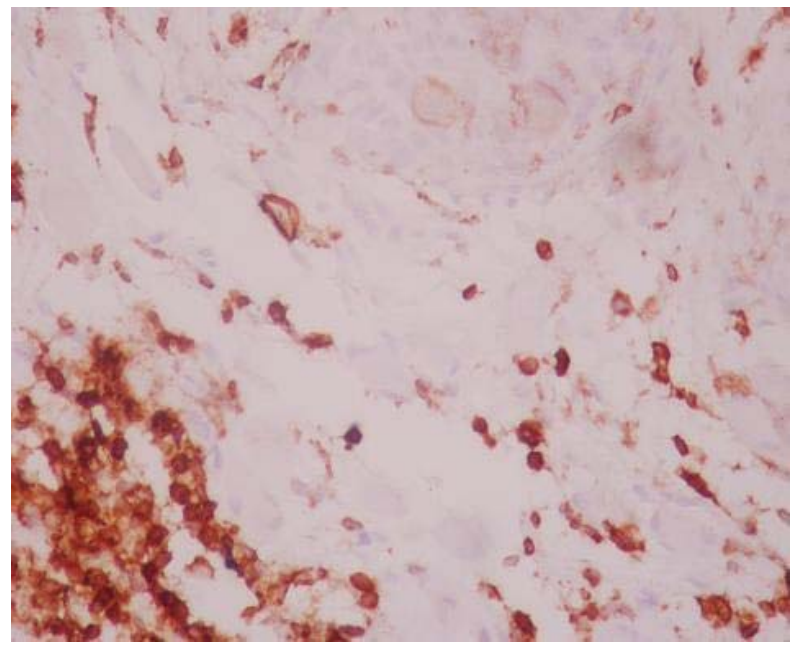

Figure 5. The tumoral cells on immunohistochemical staining were negative for Leucocyte-Common-Antigen (LCA).

The World Health Organization (WHO) has classified NPC into 3 categories:

- WHO-1 is defined as well-to-moderately differentiated squamous or transitional cell carcinoma with keratin production.

- WHO-2 is nonkeratinizing carcinoma.

- WHO-3 is undifferentiated carcinoma.

Most cases of NPC seen in Southeast Asia belong to WHO-3 (undifferentiated carcinoma). In southern China, the most common primary cancer that metastasizes to the orbit is nasopharyngeal carcinoma (30.34\%), followed by lung cancer and liver cancer [5]. There is no exact information about the most common type of NPC in Syria.

Although NPC commonly metastasizes to cervical lymph nodes, orbital metastases are rare. Most cases of orbital involvement are through direct invasion typically 
via the cavernous sinus into the apex causing proptosis and muscle paralysis. [2] less commonly, the direct spread may be via the nasolacrimal duct and in such cases epiphora is common [6].

The relationship between NPC and Epstein-Barr virus was first observed in 1966, when the sera of patients with the cancer were found to show precipitating antibodies against cells infected with the virus [7]. Later studies revealed elevated levels of IgG and IgA antibodies directed against components of Epstein-Barr virus in patients with NPC [8]. The high antibody titres to EBV antigens in NPC patients can serve as a marker for the diagnosis of NPC especially if the patients have signs of NPC but no NPC is seen during endoscopic examination. However, elevated EBV titers may also be associated with other disease entities, such as sinonasal undifferentiated carcinoma (SNUC), sinonasal lymphoma, and tongue cancer.(MAL) Circulating EBV-DNA has been shown to improve prognostication and monitoring of NPC patients [9].

Orbital metastases, even with newer diagnostic techniques can be difficult to diagnose. [10] in a study, showed that either CT or MRI provide essential information in documenting orbital invasion and determining the pathway of tumor spread [11].

An immunohistochemical panel of poorly differentiated orbital metastases is helpful in the identification of the primary tumor site. In a study, Immunohistochemistry was performed to detect cytokeratin (CK) 7, CK 20, thyroid transcription factor-1 (TTF-1), BRST1, BRST2, carcinoembryonic antigen (CEA) and prostate-specific antigen (PSA) in seven cases of poorly differentiated orbital metastases. And the association of seven markers with the patient's clinical history allowed for the positive identification of the primary tumor in the majority of these cases [12].

The aim in treating orbital metastases is to relieve discomfort. Radiotherapy is the mainstay treatment for the disease [9]. Surgical removal of the mass is not recommended. Prognosis is poor, because these patients are usually at an advanced stage of the disease. In one report, the median survival time was 1.3 years, and the two-year survival rate is $27 \%$ [13].

\section{Conclusions}

Although NPC, rarely metastases to the orbit, but should be considered in every rapidly progressing proptosis, especially in elderly.

Imaging and immunohistochemichal panel can be helpful in differentiating and rule out of other diagnoses.

\section{REFERENCES}

[1] S. M. Ahmad and B. Esmaeli, "Metastatic Tumors of the
Orbit and Ocular Adnexa," Current Opinion in Ophthalmology, Vol. 18, No. 5, 2007, pp. 405-413. doi:10.1097/ICU.0b013e3282c5077c

[2] C. C. Liaw, Y. S. Ho and K. K. Ng, "Nasopharyngeal Carcinoma Presenting as a Retro-Orbital Mass-Report of Three Cases," Otolaryngology-Head and Neck Surgery, Vol. 103, No. 5, 1990, pp. 825-828.

[3] O. Oworu, P. Kyle and R. Morton, "Metastatic Esophageal Carcinoma Presenting as a Lacrimal Gland Tumour," British Journal of Ophthalmology, Vol. 88, No. 7, 2004, pp. 972-973. doi:10.1136/bjo.2003.034322

[4] H. M. Moss, "Expanding Lesions of the Orbit. A Clinical Study of 230 Consecutive Cases," American Journal of Ophthalmology, Vol. 54, 1962, pp. 761-770.

[5] J.-H. Yan and S. Gao, "Metastatic Orbital Tumors in Southern China during an 18-Year Period," Graefe's Archive for Clinical and Experimental Ophthalmology, Vol. 249, No. 9, 2011, pp. 1387-1393. doi:10.1007/s00417-011-1660-6

[6] S. Amrith, "Antero-Medial Orbital Masses Associated with Nasopharyngeal Carcinoma,” Singapore Medical Journal, Vol. 43, No. 2, 2002, pp. 97-99

[7] L. J. Old, E. A. Boyse, H. F. Oettgen, E. Dé-Harven, G. Geering, B. Williamson, et al., "Precipitating Antibody in Human Serum to an Antigen Present in Cultured Burkitt's Lymphoma Cells,” Proceedings of the National Academy of Sciences of the USA, Vol. 56, No. 6, 1966, pp. 16991704. doi:10.1073/pnas.56.6.1699

[8] M. A. Vasef, A. Ferlito and L. M. Weiss, "Nasopharyngeal Carcinoma, with Emphasis on Its Relationship to Epstein-Barr Virus,” Annals of Otology, Rhinology and Laryngology, Vol. 106, No. 4, 1997, pp. 348-356.

[9] S. Secondino, M. Zecca, et al., "T-Cell Therapy for EBV-Associated Nasopharyngeal Carcinoma: Preparative Lymphodepleting Chemotherapy Does Not Improve Clinical Results,” Annals of Oncology, Vol. 23, No. 2, 2012, pp. 435-441.

[10] D. H Chara, T. Millerb and S. Kroll, “Orbital Metastases: Diagnosis and Course,” British Journal of Ophthalmology, Vol. 81, No. 5, 1997, pp. 386-390. doi:10.1136/bjo.81.5.386

[11] C. B. Luo, M. M. Teng, S. S. Chen, J. F. Lirng, W. Y. Guo and T. Chang, "Orbital Invasion in Nasopharyngeal Carcinoma: Evaluation with Computed Tomography and Magnetic Resonance Imaging," Chinese Medical Journal (Taipei), Vol. 61, No. 7, 1998, pp. 382-388.

[12] K. D. Godeiro, A. N. Odashiro, D. N. Odashiro, B. F. Fernandes, M. N. Burnier Jr. and S. Callejo, "Immunohistochemical Panel of Undifferentiated Orbital Metastatic Carcinomas,” Orbit, Vol. 26, No. 2, 2007, pp. 101106. doi:10.1080/01676830600977608

[13] A. P. Ferry and R. L. Font, "Carcinoma Metastatic to the Eye and the Orbit I: A Clinicopathologic Study of 227 Cases," Archives of Ophthalmology, Vol. 92, No. 4, 1974, pp. 276-286. doi:10.1001/archopht.1974.01010010286003 